\title{
Exploration of Linguistic Adaption Theory in Scientific English Vocabulary Teaching
}

\author{
Donghua Li
}

Xi'an Fanyi University, Xi'an Shaanxi, 710105, China

\author{
Keywords: Scientific English, Vocabulary Teaching, Linguistic Adaption Theory
}

\begin{abstract}
Scientific English vocabulary teaching is an important component in scientific English teaching, which is also the key and difficult teaching points. How to improve the students' understanding and abilities to master the scientific English vocabularies is the issue that college English teachers should emphasize. From the perspective of linguistic adaption theory, the paper makes some analysis. Combined with the linguistic adaption theory, it makes reform and innovation of the scientific English vocabulary teaching, so as to enhance the integration of teaching activity and students, further to promote students to learn and master scientific English. Therefore, this paper takes the linguistic adaption theory of scientific English vocabulary teaching as the research subject, hoping to offer corresponding theoretical guidance for the optimized development of scientific English vocabulary teaching.
\end{abstract}

\section{Introduction}

During recent years, in the aspect of scientific English vocabulary teaching, in order to form systematic recognition of the scientific English teaching and lead the comprehensive and optimized development of scientific English teaching activity, relevant researchers have made systematic researches on the scientific English vocabulary teaching from the linguistic cognition theory, pragmatics and cognitive lexicography, which has plays an important role in promoting the reform and innovation of scientific English vocabulary teaching. However, it should be realized that, there are still many problems of scientific English vocabulary teaching waiting to be efficiently solved. For instance, how could the students and teachers be guided to realize the conversion of unknown vocabularies; how could the scientific English vocabularies be properly applied in the scientific context, etc. Therefore, this paper makes researches on the scientific English vocabulary teaching from the perspective of linguistic adaption theory, with the aim of getting further understanding about the scientific English vocabulary teaching, and then offering corresponding theoretical guidance for the reform and innovation of teaching activities.

\section{Linguistic Adaption Theory and Scientific English Vocabulary Teaching}

Based on the language theory of Verschueren, linguistic adaption theory is considered as a quite important content, which is the foundation and core of all research work. The linguistic adaption theory mainly analyzes the interpretive language. Besides, it makes deepened interpretation from the perspective of language selection and adaption during the researching process. In the researching system of Verschueren, language users select languages after they are influenced by the interaction between consciousness and linguistic structure while they actually use languages. The process of language selection of users show certain dynamic characteristics. Looking from this perspective, as the reason why language users can consistently make selection while actually applying the languages, that's because the languages are adaptable and negotiable. Some languages will also perform the variable characteristics. Therefore, during the process of studying the linguistic phenomenon, researchers should master the correlation among the linguistic adaptability, negotiability and variability. Combined with the interactive relationship among several characteristics, researchers 
should make detailed interpretation of the dynamic variability, generativity and process. Therefore, the linguistic adaption theory could be applied to the scientific English vocabulary teaching. Combined with the dynamic adaption of scientific English vocabulary, the teaching guidance activity can be optimized and innovated, further to improve the scientific nature of teaching and guarantee the overall learning effects of students.

During the actual research and application process, the linguistic adaption theory can play certain guiding role. To introduce the linguistic adaption theory into the English teaching activity, it can reveal the dynamic adaption of scientific English education and learning of English teachers and students. Moreover, it can depict their internal ability to dynamically adapt to the scientific English vocabularies, further to guide students to enhance their recognition and understandings about the scientific English vocabularies, and then help students to complete their construction of scientific English vocabulary bank. To improve the students' ability to apply the scientific English vocabularies in teaching activity, teachers should implement corresponding guidance over students in different contexts, so as to let students make dynamic selection and adaption while using vocabularies in different contexts, and then guarantee the effects to apply scientific English vocabularies[1]. From the perspective of linguistic adaption theory, the scientific English vocabulary teaching actually takes students' ability to select and adapt scientific English vocabularies as the orientation. With the help of selection and interaction, it can cultivate the students' ability to apply language during the entire teaching activity. Just based on that, combined with the guidance of linguistic adaption theory, it can construct the core ideology of scientific English vocabulary during the teaching process. In addition, to strengthen the vocabulary of students, it cannot only let students understand vocabularies simply, but also perceive the external things besides vocabularies. On the basis of learning the scientific English vocabularies, students can form the ability of scientific English language, further to lay foundation for the professional English qualities. Based on this, in the teaching reform activity, teachers can offer corresponding guidance for scientific English vocabulary teaching activity with the help pf linguistic adaption theory, and also make reform and innovation of scientific English vocabulary teaching. With the help of scientific and reasonable teaching activity, it can indeed give full play to the important role of scientific English vocabulary teaching, further to lay a solid foundation for strengthening the comprehensive qualities of students.

\section{The Enlightenment of Linguistics Adaption Theory to Scientific English Vocabulary Teaching}

From the perspective of linguistics adaption theory, it studies the scientific English vocabulary teaching. According to the guidance of linguistics adaption theory, teachers should focus on cultivating students' ability of vocabularies while developing the scientific English teaching, show the generation and adaption process of scientific English vocabularies to students. On the basis of language selection and adaption, students can form deep understandings about the scientific English vocabularies, further to reinforce the priming effect of scientific English vocabularies, activate their ability to form words and analyze semantics while learning the scientific English vocabularies, further to form better ability to apply the vocabularies. In this research work, combined with the guidance of linguistic adaption theory, it suggests that teachers should focus on cultivating students' ability of scientific English negotiability, variability and adaptability under the frame of cultivating their vocabulary ability, let them form systematic understandings about the negotiability, variability and adaptability of scientific English vocabulary, and then create good conditions for cultivating linguistic competence.

\subsection{Variable Vocabulary Competence Cultivating Teaching}

In the scientific English vocabulary teaching, in order to cultivate the vocabulary competence of students, they should master deeper knowledge of vocabularies, so as to create conditions for the scientific English linguistic competence later. Meanwhile, in the linguistic adaption theory system, vocabulary competence is actually an important component of people's cognitive competence. With 
the help of vocabulary competence, people can analyze the transferred meanings related to vocabularies, further to obtain more information. After making systematic analyze on the scientific English vocabularies, it discovers that, it usually applies vocabularies to represent professional knowledge and relevant concepts of the scientific English. Therefore, some vocabularies usually have the transferred meanings in English, which turn to be special meanings in the area of scientific English. In another word, the variability of vocabulary is quite common in the linguistic system of scientific English, which shows that the scientific English vocabularies are actually characterized of selectivity. During the process of transforming from the original meaning to the transferred meaning or metaphorical meaning, it can be selected from many perspectives. Therefore, from the perspective of variability, as a matter of fact, the scientific English vocabulary teaching activity developed by teachers is to guide students to make reasonable dynamic selections among those optional and possible words. In addition, they can carry out corresponding activities on selected words. The main objective of teaching is to let students master the internal correlation of different items during the process of vocabulary variability with the help of system, further to master the law of variability, guess the transferred meaning and metaphorical meaning of related scientific English vocabulary, so as to guarantee the well cultivation of students' ability to apply scientific English variable vocabularies.

For instance, in the scientific English, "chamber" is the variation and transformation of the original meaning, which generates different semantics. To make detailed analysis on "chamber", the original meaning refers to the "cavity" of body. Under the influence of the variability of scientific English vocabulary, it makes proper extension and generates many different meanings in different scopes. As the external representation of "mysterious space”, it can be also applied to some scientific English vocabularies, such as "brake chamber", "chamber" and "model tank". There is certain relationship between the variability of these scientific English vocabularies and the original meaning of "chamber". It is an extended vocabulary network system centering on "chamber". Therefore, during the scientific English vocabulary teaching, it should strengthen the cultivation of students' competence of variable vocabularies. Students should be guided to form systematic understandings about the relationship among the original meaning, transferred meaning and metaphorical meaning, further to strengthen their understandings about scientific English vocabularies. Combined with the variability law among the original meaning, transferred meaning and metaphorical meaning, it can guarantee the realization of understandings about the scientific English vocabularies and correct application.

\subsection{Negotiable Vocabulary Competence Cultivating Teaching}

From the variability presented by the scientific English vocabularies, the interaction of the original meaning, transferred meaning and metaphorical meaning has formed a complicated vocabulary network system. It requires that English teachers cannot guide students to learn or understand the scientific English vocabularies according to fixed patterns, but should let students form clear recognition of the scientific English vocabularies on the basis of mastering the principle and strategy of vocabularies, with the help of quite flexible means. Meanwhile, from the perspective of students, the scientific English vocabulary equals to polysemy and new word. Thus students have certain difficulties in leaning and exploring vocabularies. Therefore, teachers should pay attention to cultivating students' ability of negotiable scientific English vocabularies, further to make extensions of the scientific English vocabularies on the basis of regular vocabularies, realize the cultivation of negotiable vocabulary competence and guarantee the correct application of the scientific English vocabularies.

For instance, the original meaning of "fault" refers to "mistake, error". However, after it is transferred to the scientific English area, the meaning has been changed, which refers to "breakdown", "failure", "fracture", etc. A radiated vocabulary system has been formed. Besides, there is certain correlation between every derivative and original meaning. However, it cannot be influenced by the original meaning, but with comparatively obvious independence. During the process of learning the scientific English vocabularies, in order to guarantee the learning effects of students, teachers should 
ask students to make analysis on the optional semantic items, combined with the dynamic changes and adaptability of vocabularies, so as to enhance students' effectiveness of learning the scientific English vocabularies. For instance, as students learn the "fault", they would firstly activate the semantic items of "fault". On that basis, combined with the dynamic changes and adaptability of vocabularies, they can activate other meanings of semantic items. The negotiability competence of the scientific English vocabularies can be properly cultivated, which has great significance for students to make deeper learning and understand the scientific English vocabularies.

\subsection{Adaptable Vocabulary Competence Cultivating Teaching}

From the perspective of vocabulary competence cultivation, in order to let students master the competence of accurately using vocabularies with the help of efficient teaching guidance, teachers have to let students make selections and judgement targeted at the English vocabularies, and also make dynamic adaptions, so as to guarantee the application of selected vocabularies to actually satisfy the demands. In the scientific English vocabulary teaching, the cultivation of students' variable and negotiable vocabulary competence has offered precondition for the adaptability teaching. The cultivation of adaptability competence has put forward new requirements for the teaching activity. Teachers need to center on students and make proper selections targeted at different scientific English vocabularies, further to enhance the application effected of scientific English vocabularies.

For instance, while teaching and interpreting the scientific English vocabulary "exhaust”, teachers need to let students know the basic meaning of "tiring, wear out". After extending the meaning, the "exhaustion" in the scientific vocabulary has also become an important semantics, which displays certain sharing features. There is certain correlation between the original meaning of the word and the extended meaning. Under that influence, during the actual application and selection process of the scientific English vocabularies, the extended meaning of "exhaust" has been fatherly extended, such as "air exhaust”, “emission”, "tail gas”, etc. During the consistently extended process, a huge distance has generated between these extended meaning and the original meaning of "exhaust". We can barely see the relationship any more. Therefore, in order to guarantee the students' leaning effects of scientific English vocabularies, they have to cultivate their competence of adaptable vocabulary and let students shorten the gap between the original meaning and extended meaning with the adaptable vocabulary competence, discover the internal correlation, further to construct the vocabulary chain and indeed increase their learning effects.

\section{Conclusions}

Above all, under the social background of booming theories of pragmatics, it can better explain the phenomenon of the scientific English vocabulary teaching by studying and analyzing it from the perspective of pragmatics, further to offer corresponding guidance for the reform and innovation of teaching. Therefore, combined with the linguistic adaption theory of the pragmatics system, it studies the scientific English vocabulary teaching. On the basis of teaching reform, teachers center on the students, guide them to form deeper understandings about the negotiability, adaptability and variability of the scientific English vocabulary teaching, promote the cultivation of English vocabulary competence, let them better apply the scientific English vocabularies and have positive influences on improving students' linguistic competence of scientific English, further to promote the comprehensive improvement of teaching quality and gradual optimization of talents cultivation.

\section{References}

[1] Luo Dijiang, Chen Yuanyuan. Researches on the Linguistic Adaption Theory of Scientific English Vocabulary Teaching, Journal of Chengdu Normal University, 2017, 33(2):67-70.

[2] Wang Chengwei. Enlightenment of The Prototype Theory on English Vocabulary Teaching, Heilongjiang Science and Technology Information, 2011(19):171-171. 
[3] Zhang Dan. Enlightenment of The Prototype Scope Theory on English Vocabulary Teaching--Prototype Exploration of English Part of Speech and Tense, Science and Technology Information, 2012(34):605+608.

[4] Wen Tong. Researches on the Guidance of Cognitive Linguistic Theory on College English Vocabulary Teaching, Youth and Society Magazine: the first half volume, 2014(8):127-128.

[5] Chen Chen. Researches on the Application of Multi-modal Vocabulary Teaching Mode in Vocabulary Teaching for Non-English Major College Students, Inner Mongolia Normal University, 2015. 